\title{
Analysis of the Finite Element Variable Penalty Method for Stokes Equations
}

\section{By Haroon Kheshgi* and Mitchell Luskin**}

\begin{abstract}
We give an error analysis of the finite element variable penalty method for Stokes equations. It is shown that the variable penalty method is of higher order than the standard penalty method.
\end{abstract}

1. Introduction. Stokes system for viscous, incompressible flow is given by

$$
\begin{array}{ll}
-\nabla \cdot \underline{D}(\mathbf{u})+\nabla p=\mathbf{f}, & x \in \Omega, \\
\nabla \cdot \mathbf{u}=0, & x \in \Omega,
\end{array}
$$

where $\mathbf{u}=\left(u_{1}, u_{2}\right)$ is velocity, $p$ is pressure, $\mathbf{f}$ is a given body force, $\Omega$ is a bounded domain in $\mathbf{R}^{2}$, and

$$
\begin{aligned}
& D_{i j}(\mathbf{u})=\left(\frac{\partial u_{i}}{\partial x_{j}}+\frac{\partial u_{j}}{\partial x_{i}}\right), \quad i, j=1,2, \\
& (\nabla \cdot \underline{\underline{D}})_{i}=\sum_{j=1}^{2} \frac{\partial}{\partial x_{j}} D_{i j}, \quad i=1,2 .
\end{aligned}
$$

The penalty method for Stokes equations replaces the continuity equation

$$
\nabla \cdot \mathbf{u}=0
$$

by the perturbed equation

$$
\nabla \cdot \mathbf{u}=-\varepsilon p, \quad x \in \Omega,
$$

where $\varepsilon>0$ is a small parameter (see the references in [3], [9]). The purpose of introducing the approximation (1.2) is that it allows the pressure variable to be eliminated from (1.1) to give the "simpler" system of equations

$$
-\nabla \cdot \underline{\underline{D}}(\mathbf{u})-\nabla\left(\frac{1}{\varepsilon} \nabla \cdot \mathbf{u}\right)=\mathbf{f}, \quad x \in \Omega
$$

In this paper, we analyze a related perturbation method, the variable penalty method, which replaces the continuity equation by

$$
\nabla \cdot \mathbf{u}=-\varepsilon \varphi_{h} p, \quad x \in \Omega,
$$

Received May 24, 1983; revised June 28, 1984 and November 14, 1984.

1980 Mathematics Subject Classification. Primary 65N30, 75D05.

${ }^{*}$ Current address: Lawrence Livermore National Laboratory, Livermore, CA 94550 . Supported by a Grant-in-Aid from Kodak Research Laboratories.

**Supported by the NSF. Grants MCS-810-1631, MCS-830-1575, and MCS-835-1080. 
where $\left|\varphi_{h}(x)\right| \leqslant 1, x \in \Omega$, and where $\varphi_{h}$ alternates sign on a grid of size $h$. This perturbation method also allows the pressure to be eliminated from (1.1) to give the system of equations

$$
-\nabla \cdot \underline{\underline{D}}(\mathbf{u})-\nabla \cdot\left(\frac{1}{\varepsilon \varphi_{h}} \nabla \cdot \mathbf{u}\right)=\mathbf{f}, \quad x \in \Omega .
$$

The penalty method is often used to eliminate the pressure unknowns from the linear system of equations which is obtained from finite element (or finite difference) discretizations of Stokes equations [4]. Similarly, the variable penalty method can be used to eliminate the pressure unknowns from finite element discretizations of Stokes equations. Recent numerical experiments have shown that the finite element variable penalty method is more accurate than the standard finite element penalty method [5], [6], [7]. The purpose of this paper is to explain these results by giving error estimates for the variable penalty method that are of higher order for the velocity than those which can be obtained for the standard penalty method. We also prove that a higher-order method for the pressure can be obtained if the computed pressure is appropriately smoothed (or post-processed).

References for known results will often be to the texts, [3] and [9], rather than to original papers (which are listed in the bibliographies of [3] and [9]).

2. Definitions and Main Results. We assume that $\Omega$ has a $C^{\infty}$ boundary, $\partial \Omega$, which is the union of an interior closed curve, $\Gamma_{1}$, and an exterior closed curve, $\Gamma_{2}$.

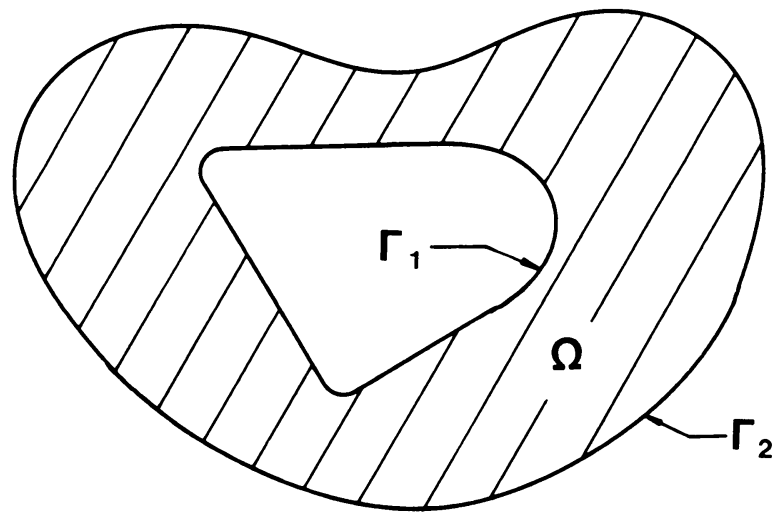

FIGURE 1

We will be interested in the boundary-value problem

$$
\begin{array}{ll}
(\underline{D}(\mathbf{u})-p \underline{I}) \mathbf{n}=\mathbf{0}, & x \in \Gamma_{1}, \\
\mathbf{u}=\mathbf{0}, & x \in \Gamma_{2},
\end{array}
$$

where $\mathbf{n}$ is the unit exterior normal to $\Gamma_{1}$. This choice of topology for $\Omega$ and this choice of boundary conditions guarantees that (1.1) has a unique smooth solution for smooth data. Thus far, we have been unable to analyze the variable penalty method when the values of the velocity are given on the entire boundary, although the variable penalty method has been tested successfully for this boundary-value problem in numerical experiments. 
For scalar-valued functions $\xi, \eta \in L^{2}(\Omega)$, we define the inner product and norm

$$
(\xi, \eta)=\int_{\Omega} \xi \eta d x, \quad\|\xi\|^{2}=(\xi, \xi) .
$$

We denote by $H^{k}(\Omega), k$ a nonnegative integer, the space of functions

$$
H^{k}(\Omega)=\left\{\xi \in L^{2}(\Omega) \mid D^{\alpha} \xi \in L^{2}(\Omega) \text { for }|\alpha| \leqslant k\right\}
$$

with norm

$$
\|\xi\|_{k}^{2}=\sum_{|\alpha| \leqslant k}\left\|D^{\alpha} \xi\right\|^{2}
$$

We wish to also extend the above definitions in the usual fashion to vector-valued functions $\mathbf{v}: \Omega \rightarrow \mathbf{R}^{2}$, and we denote the resulting spaces by $L^{2}(\Omega)^{2}, H^{k}(\Omega)^{2}$, etc. For $\mathbf{v}: \Omega \rightarrow \mathbf{R}^{2}, \xi: \Omega \rightarrow \mathbf{R}$, and $l, m \geqslant 0$ integers we define the norm

$$
\|\langle\mathbf{v}, \xi\rangle\|_{l, m}^{2}=\|\mathbf{v}\|_{l}^{2}+\|\xi\|_{m}^{2}, \quad \mathbf{v} \in H^{l}(\Omega)^{2}, \xi \in H^{m}(\Omega) .
$$

Finally, it will be useful to define the spaces

$$
H_{b}^{1}(\Omega)=\left\{\mathbf{v} \in H^{1}(\Omega)^{2} \mid \mathbf{v}=\mathbf{0} \text { on } \Gamma_{2}\right\},
$$

and

$$
\mathscr{V}_{b}=\left\{\langle\mathbf{v}, g\rangle \mid \mathbf{v} \in H_{b}^{1}(\Omega) \text { and } g \in L^{2}(\Omega)\right\} .
$$

We next define the continuous bilinear forms

$$
\begin{aligned}
& a(\mathbf{w}, \mathbf{v})=(\underline{\underline{D}}(\mathbf{w}), \underline{\underline{D}}(\mathbf{v})), \quad \mathbf{w}, \mathbf{v} \in H_{b}^{1}(\Omega), \\
& b(r, \mathbf{v})=(r, \nabla \cdot \mathbf{v}), \quad r \in L^{2}(\Omega), \mathbf{v} \in H_{b}^{1}(\Omega), \\
& B(\langle\mathbf{w}, r\rangle,\langle\mathbf{v}, q\rangle)=a(\mathbf{w}, \mathbf{v})-b(r, \mathbf{v})-b(q, \mathbf{w}), \quad\langle\mathbf{w}, r\rangle,\langle\mathbf{v}, q\rangle \in \mathscr{V}_{h} .
\end{aligned}
$$

Let $c_{0}>0$ be such that

$$
B(\langle\mathbf{w}, r\rangle,\langle\mathbf{v}, q\rangle) \leqslant c_{0}\|\langle\mathbf{w}, r\rangle\|_{1,0}\|\langle\mathbf{v}, q\rangle\|_{1,0}, \quad\langle\mathbf{w}, r\rangle,\langle\mathbf{v}, q\rangle \in \mathscr{V}_{b} .
$$

Since the divergence operator is a map from $H_{b}^{1}(\Omega)$ onto $L^{2}(\Omega)$, the analysis in [3, p. 50] shows that there exists a positive constant, $c_{1}$, such that

$$
\sup _{\langle\mathbf{v}, q\rangle \in \mathscr{V}_{b}} \frac{B(\langle\mathbf{w}, r\rangle,\langle\mathbf{v}, q\rangle)}{\|\langle\mathbf{v}, q\rangle\|_{1,0}} \geqslant c_{1}\|\langle\mathbf{w}, r\rangle\|_{1,0}, \quad\langle\mathbf{w}, r\rangle \in \mathscr{V}_{b} .
$$

Now suppose that $\mathbf{f} \in L^{2}(\Omega)^{2}$. It then follows from (2.1) [3], [9] that there exists a unique $\langle\mathbf{u}, p\rangle \in \mathscr{V}_{b}$ so that

$$
B(\langle\mathbf{u}, p\rangle,\langle\mathbf{v}, q\rangle)=(\mathbf{f}, \mathbf{v}), \quad\langle\mathbf{v}, q\rangle \in \mathscr{V}_{b} .
$$

We call $\langle\mathbf{u}, p\rangle \in \mathscr{V}_{b}$ the solution to (1.1).

To approximate the problem (2.2) we let $h$ denote a discretization parameter tending to zero, and we let $X_{h}$ and $M_{h}$ be finite-dimensional subspaces such that

$$
X_{h} \subset H_{b}^{1}(\Omega) \text { and } M_{h} \subset L^{2}(\Omega) .
$$

Also, let

$$
\mathscr{V}_{b h}=X_{h} \times M_{h}
$$

We assume that $c_{1}$ can also be chosen independent of $h$ so that

$$
\sup _{\left\langle\mathbf{v}_{h}, q_{h}\right\rangle \in \mathscr{V}_{h h}} \frac{B\left(\left\langle\mathbf{w}_{h}, r_{h}\right\rangle,\left\langle\mathbf{v}_{h}, q_{h}\right\rangle\right)}{\left\|\left\langle\mathbf{v}_{h}, q_{h}\right\rangle\right\|_{1,0}} \geqslant c_{1}\left\|\left\langle\mathbf{w}_{h}, r_{h}\right\rangle\right\|_{1,0}, \quad\left\langle\mathbf{w}_{h}, r_{h}\right\rangle \in \mathscr{V}_{h h} .
$$



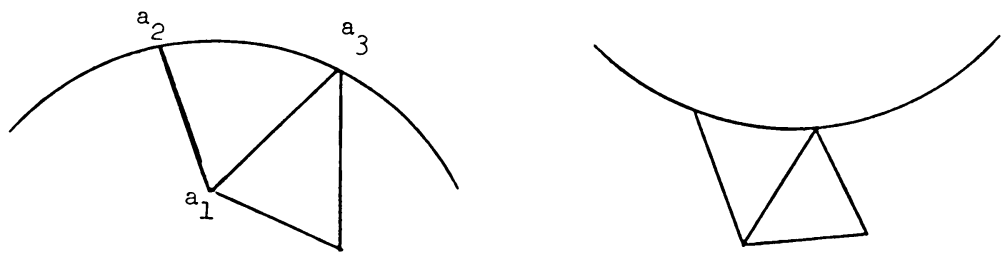

Figure 2

The classical finite element solution to Stokes problem is $\left\langle\mathbf{u}_{h}, p_{h}\right\rangle \in \mathscr{V}_{h h}$ such that

$$
B\left(\left\langle\mathbf{u}_{h}, p_{h}\right\rangle,\left\langle\mathbf{v}_{h}, q_{h}\right\rangle\right)=\left(\mathbf{f}, \mathbf{v}_{h}\right), \quad\left\langle\mathbf{v}_{h}, q_{h}\right\rangle \in \mathscr{V}_{h h} .
$$

Many of the classical finite element spaces $X_{h}$ and $M_{h}$, properly modified at the boundary, can be shown to satisfy the hypothesis (2.3). For example, let $\Omega$ be an annulus and let $\left\{\mathscr{T}_{h}\right\}$ be a uniformly regular family of triangulations of $\Omega$ such that the triangles bordering the outer boundary are pie-shaped and such that the triangles bordering the inner boundary have curved boundaries as shown in Figure 2 .

We then set $M_{h}$ to be the space of functions which are piecewise constant with respect to the triangulation, $\mathscr{T}_{h}$. We further set $X_{h}$ to be the space of continuous functions, $\mathbf{w}_{h}(x)$, which are piecewise quadratic with respect to the triangles which do not border the outer boundary. For triangles, $K \in \mathscr{T}_{h}$, which border the outer boundary (see Figure 2), we restrict $\mathbf{w}_{h}(x)$ to be quadratic in the inscribed triangle $K^{\prime} \subset K$ with straight edges and vertices $\left[a_{1}, a_{2}, a_{3}\right]$ and to be zero in $K-K^{\prime}$. It can be shown [2] that there exists a positive constant, $c$, independent of $h$, such that for $\mathbf{w}(x) \in H_{b}^{1}(\Omega) \cap H^{2}(\Omega)^{2}$,

$$
\inf _{\mathbf{w}_{h} \in X_{h}}\left\|\mathbf{w}-\mathbf{w}_{h}\right\|_{1}<\operatorname{ch}\|\mathbf{w}\|_{2} .
$$

The argument in [3, p. 76] can then be used to prove (2.3). Clearly, this argument can be extended to more general domains, $\Omega$, such as domains which are diffeomorphic to an annulus and which have a convex outer boundary, $\Gamma_{2}$, and to many of the other classical finite element spaces $X_{h}$ and $M_{h}$ which give stable approximations of Stokes equations. It follows from (2.3) that (2.4) has a unique solution $\left\langle\mathbf{u}_{h}, p_{h}\right\rangle \in \mathscr{V}_{b h}$ and

$$
\left\|\left\langle\mathbf{u}-\mathbf{u}_{h}, p-p_{h}\right\rangle\right\|_{1,0} \leqslant\left(1+\frac{c_{0}}{c_{1}}\right) \inf _{\left\langle\mathbf{v}_{h}, q_{h}\right\rangle \in \mathscr{V}_{b h}}\left\|\left\langle\mathbf{u}-\mathbf{v}_{h}, p-q_{h}\right\rangle\right\|_{1,0} .
$$

The penalty method is to approximate the solution to (2.4) by the solution $\left\langle\mathbf{u}_{h \varepsilon}, p_{h \varepsilon}\right\rangle \in \mathscr{V}_{b h}$ to

(2.6) $B\left(\left\langle\mathbf{u}_{h \varepsilon}, p_{h \varepsilon}\right\rangle,\left\langle\mathbf{v}_{h}, q_{h}\right\rangle\right)-\varepsilon\left(p_{h \varepsilon}, q_{h}\right)=\left(\mathbf{f}, \mathbf{v}_{h}\right), \quad\left\langle\mathbf{v}_{h}, q_{h}\right\rangle \in \mathscr{V}_{b h}$,

where $\varepsilon>0$ is a small parameter. It follows that

$$
b\left(q_{h}, \mathbf{u}_{h \varepsilon}\right)=-\varepsilon\left(p_{h \varepsilon}, q_{h}\right), \quad q_{h} \in M_{h} .
$$

We can define the discrete divergence operator, $\nabla_{h} \cdot: X_{h} \rightarrow M_{h}$ by $\nabla_{h} \cdot \mathbf{w}_{h}=r_{h}$, where

$$
b\left(\mathbf{w}_{h}, q_{h}\right)=\left(q_{h}, \nabla_{h} \cdot \mathbf{w}_{h}\right)=\left(q_{h}, r_{h}\right), \quad q_{h} \in M_{h},
$$

and then (2.7) is equivalent to

$$
\nabla_{h} \cdot \mathbf{u}_{h \varepsilon}=-\varepsilon p_{h \varepsilon}
$$


We can thus use (2.8) to eliminate $p_{h \varepsilon}$ from (2.6) to obtain the finite element approximation of (1.3)

$$
a\left(\mathbf{u}_{h \varepsilon}, \mathbf{v}_{h}\right)+\frac{1}{\varepsilon}\left(\nabla_{h} \cdot \mathbf{u}_{h \varepsilon}, \nabla_{h} \cdot \mathbf{v}_{h}\right)=\left(\mathbf{f}, \mathbf{v}_{h}\right), \quad \mathbf{v}_{h} \in X_{h} .
$$

If the pressure space, $M_{h}$, is a space of discontinuous, piecewise polynomials, then $\nabla_{h} \cdot \mathbf{v}_{h}$ can be constructed locally on the elements. Thus, we see that the penalty method can be used to eliminate the pressure variables from the discrete equations to reduce the size of the system of linear equations to be solved as well as the bandwidth, and the pressure variables need never be included in the assembled matrix problem [4].

The following approximation theorem is now well-known (although our proof may be new).

THEOREM 1 [3]. If $|\varepsilon| \leqslant c_{1} / 2$, then the penalty method (2.6) has a unique solution $\left\langle\mathbf{u}_{h \varepsilon}, p_{h \varepsilon}\right\rangle \in \mathscr{V}_{h h}$, and we have the estimate that

$$
\begin{aligned}
\left\|\left\langle\mathbf{u}-\mathbf{u}_{h \varepsilon}, p-p_{h \varepsilon}\right\rangle\right\|_{1,0} \leqslant & \left(1+\frac{2\left(c_{0}+|\varepsilon|\right)}{c_{1}}\right) \inf _{\left\langle\mathbf{w}_{h}, r_{h}\right\rangle \in \mathscr{V}_{h h}}\left\|\left\langle\mathbf{u}-\mathbf{w}_{h}, p-r_{h}\right\rangle\right\|_{1,0} \\
& +\frac{2|\varepsilon|}{c_{1}}\|p\| .
\end{aligned}
$$

However, we must consider round-off error in addition to approximation error, since the linear system (2.9) must be solved in practice by digital computers in floating-point arithmetic, and it is observed in practice that the round-off error for the solution of these linear systems by Gaussian elimination is $O(1 / \varepsilon)$ [4], [5], [6], [7]. This can be explained theoretically by noting that the condition number of the linear system $(2.9)$ is $O(1 / \varepsilon)$. Hence, the penalty method is sufficiently accurate for only a restricted range of the parameter, $\varepsilon$. Further, it may not be possible to achieve sufficient accuracy for any $\varepsilon$ with the penalty method for some problems, especially if one is using a computer with a small word length.

The variable penalty method is to approximate the solution to (2.4) by the solution $\left\langle\mathbf{u}_{h \varepsilon}, p_{h \varepsilon}\right\rangle \in \mathscr{V}_{b h}$ to

$$
B\left(\left\langle\mathbf{u}_{h \varepsilon}, p_{h \varepsilon}\right\rangle,\left\langle\mathbf{v}_{h}, q_{h}\right\rangle\right)-\varepsilon\left(\varphi_{h} p_{h \varepsilon}, q_{h}\right)=\left(\mathbf{f}, \mathbf{v}_{h}\right), \quad\left\langle\mathbf{v}_{h}, q_{h}\right\rangle \in \mathscr{V}_{b h},
$$

where $\varepsilon$ is a small parameter and $\varphi_{h}$ is a measurable function satisfying

$$
\left|\varphi_{h}(x)\right| \leqslant 1, \quad x \in \Omega .
$$

We will show that the approximation (2.11) is of higher order than (2.6) if there exists $c_{2}>0$, independent of $h$, such that

$$
\int_{\Omega} \varphi_{h} \xi d x \leqslant c_{2} h \int_{\Omega}|\nabla \xi| d x
$$

for all functions $\xi$ satisfying

$$
\int_{\Omega}|\nabla \xi| d x<\infty
$$

It follows by arguments similar to those for the standard penalty method that

$$
b\left(\mathbf{u}_{h \varepsilon}, q_{h}\right)=-\varepsilon\left(\varphi_{h} p_{h \varepsilon}, q_{h}\right), \quad q_{h} \in M_{h},
$$


so

$$
\nabla_{h} \cdot \mathbf{u}_{h \varepsilon}=-\varepsilon \mathbf{P}_{h}\left(\varphi_{h} p_{h \varepsilon}\right),
$$

where $\mathbf{P}_{h}: L^{2}(\Omega) \rightarrow M_{h}$ is the standard $L^{2}(\Omega)$-projection operator. We shall assume, henceforth, that

$$
\varphi_{h} q_{h} \in M_{h} \text { for } q_{h} \in M_{h}
$$

so

$$
\nabla_{h} \cdot \mathbf{u}_{h \varepsilon}=-\varepsilon \varphi_{h} p_{h \varepsilon} \cdot
$$

Thus, we can use (2.16) to eliminate the pressure from (2.11) to obtain the following finite element approximation of (1.5)

$$
a\left(\mathbf{u}_{h \varepsilon}, \mathbf{v}_{h}\right)+\left(\frac{1}{\varepsilon \varphi_{h}} \nabla_{h} \cdot \mathbf{u}_{h \varepsilon}, \nabla_{h} \cdot \mathbf{v}_{h}\right)=\left(\mathbf{f}, \mathbf{v}_{h}\right), \quad \mathbf{v}_{h} \in X_{h} .
$$

In our numerical experiments [5], [6], [7], we have used discontinuous, piecewise polynomial functions of fixed degree with respect to a triangulation of size $h$ for $M_{h}$, and $\varphi_{h}$ has been a piecewise constant function with respect to that triangulation [5], [6], [7]. Obviously, (2.15) is satisfied in this case and the variable penalty method is no more difficult to implement than the standard penalty method. There are many ways to construct $\varphi_{h}$ so that (2.13) is valid. A simple choice for $\varphi_{h}$ on a rectangular grid is to let $\varphi_{h}=1$ or -1 on alternating rows (see Figure 3).

In Section 3, we prove a result for the variable penalty method which is identical to the result for the standard penalty method, which we have given as Theorem 1 .

THEOREM 2. If $|\varepsilon| \leqslant c_{1} / 2$, then the variable penalty method (2.11) has a unique solution $\left\langle\mathbf{u}_{h \varepsilon}, p_{h \varepsilon}\right\rangle \in \mathscr{V}_{b h}$, and we have the estimate

$$
\begin{aligned}
\left\|\left\langle\mathbf{u}-\mathbf{u}_{h \varepsilon}, p-p_{h \varepsilon}\right\rangle\right\|_{1,0} \leqslant & \left(1+\frac{2\left(c_{0}+|\varepsilon|\right)}{c_{1}}\right) \inf _{\left\langle\mathbf{w}_{h}, r_{h}\right\rangle \in \mathscr{V}_{h h}}\left\|\left\langle\mathbf{u}-\mathbf{w}_{h}, p-r_{h}\right\rangle\right\|_{1,0} \\
& +\frac{2|\varepsilon|}{c_{1}}\|p\| .
\end{aligned}
$$

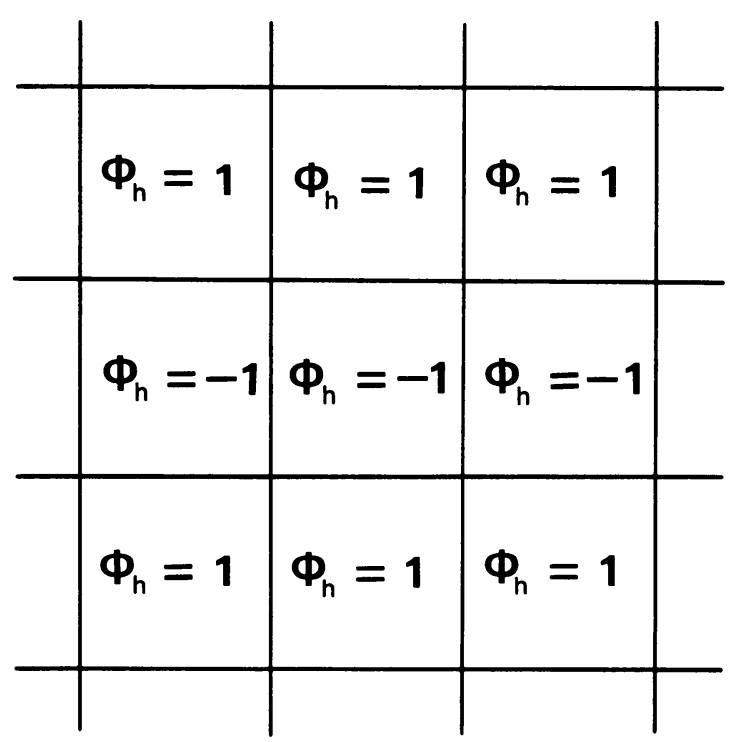

Figure 3 
To demonstrate that the variable penalty method is of higher order than the penalty method, we estimate the error in the velocity in $L^{2}(\Omega)^{2}$. We have assumed that the boundary of $\Omega, \partial \Omega=\Gamma_{1} \cup \Gamma_{2}$, is smooth so that there exists $c_{3}>0$ such that if $\langle\mathbf{v}, q\rangle \in L^{2}(\Omega)^{2} \times H^{1}(\Omega)$, then the unique solution $\langle\mathbf{z}, m\rangle \in \mathscr{V}_{b}$ to the problem

$$
B(\langle\mathbf{w}, r\rangle,\langle\mathbf{z}, m\rangle)=(\mathbf{w}, \mathbf{v})+(r, q), \quad\langle\mathbf{w}, r\rangle \in \mathscr{V}_{b},
$$

has the property that $\langle\mathbf{z}, m\rangle \in H^{2}(\Omega)^{2} \times H^{1}(\Omega)$ and $\langle\mathbf{z}, m\rangle$ satisfies the a priori estimate

$$
\|\langle\mathbf{z}, m\rangle\|_{2,1} \leqslant c_{3}\|\langle\mathbf{v}, q\rangle\|_{0,1}
$$

We also now assume that there exists $c_{4}>0$, independent of $h$, and $r \geqslant 1$, such that our subspaces $X_{h}$ and $M_{h}$ satisfy the approximation property that for each $\langle\mathbf{v}, q\rangle \in \mathscr{V}_{b} \cap H^{r+1}(\Omega)^{2} \times H^{r}(\Omega)$ there exists $\left\langle\mathbf{v}_{h}, q_{h}\right\rangle \in \mathscr{V}_{b h}$ such that

$$
\left\|\left\langle\mathbf{v}-\mathbf{v}_{h}, q-q_{h}\right\rangle\right\|_{1,0} \leqslant c_{4} h^{s}\|\langle\mathbf{v}, q\rangle\|_{s+1, s}, \quad 0 \leqslant s \leqslant r .
$$

The piecewise constant space, $M_{h}$, and the continuous, piecewise quadratic space, $X_{h}$, described above satisfies (2.20) with $r=1$. Isoparametric spaces which satisfy the zero boundary conditions on approximate domains and the associated analysis [2] are required to obtain higher-order approximation properties for curved boundaries.

In the following, it will be convenient to use a generic positive constant, $c$, which is independent of $h$ and $\varepsilon$, but which can change from equation to equation. In Section 3, under the above hypotheses, we will prove the following result.

ThEOREM 3. Let $|\varepsilon| \leqslant c_{1} / 2$. There exists a positive constant, $c$, such that if the solution $\langle\mathbf{u}, p\rangle$ to $(2.4)$ satisfies $\langle\mathbf{u}, p\rangle \in H^{r+1}(\Omega)^{2} \times H^{r}(\Omega)$, then

$$
\left\|\mathbf{u}-\mathbf{u}_{h \varepsilon}\right\| \leqslant c\left(|\varepsilon| h\|\langle\mathbf{u}, p\rangle\|_{2,1}+|\varepsilon|^{2}\|p\|+h^{r+1}\|\langle\mathbf{u}, p\rangle\|_{r+1, r}\right) .
$$

To achieve a higher-order method for the pressure we define

$$
K(x)=K\left(x_{1}, x_{2}\right)= \begin{cases}(2 h)^{-2}, & \text { if }\left|x_{i}\right| \leqslant h, i=1,2 \\ 0, & \text { otherwise }\end{cases}
$$

Let $\tilde{\Omega} \subset \subset \Omega$. Then

$$
K * p_{h \varepsilon}(x)=\int_{\Omega} K(x-y) p_{h \varepsilon}(y) d y, \quad x \in \tilde{\Omega},
$$

is well-defined for $\sqrt{2} h<\operatorname{dist}(\tilde{\Omega}, \partial \Omega)$. We will prove in Section 5 under several additional hypotheses which are valid for finite element methods, when the triangulation is regular on $\tilde{\Omega}$, that if $\Omega_{1} \subset \subset \tilde{\Omega},\langle\mathbf{u}, p\rangle \in H^{r+2}(\Omega)^{2} \times H^{r+1}(\Omega)$, and $h$ is sufficiently small, then

$$
\left\|p-K * p_{h \varepsilon}\right\|_{\Omega_{1}}
$$

$$
\leqslant c\left(|\varepsilon| h\|\langle\mathbf{u}, p\rangle\|_{2,2}+|\varepsilon|^{2}\|p\|+h^{2}\|p\|_{2}+h^{r+1}\|\langle\mathbf{u}, p\rangle\|_{r+2, r+1}\right),
$$

where $\|\xi\|_{\Omega_{1}}^{2}=\int_{\Omega_{1}} \xi^{2} d x$. 
These estimates for the variable penalty method demonstrate that it can be expected to achieve a desired accuracy at a larger value of $|\varepsilon|$ than is achieved by the standard penalty method. Since it is equally sensitive to round-off error [5], [6], [7], the variable penalty method achieves a desired accuracy for a wider range of $|\varepsilon|$. Further, for a given triangulation, the desired accuracy may be obtainable by the variable penalty method and may not be obtainable by the standard penalty method.

3. Main Estimates. Let

$$
\begin{array}{r}
B_{\varepsilon}(\langle\mathbf{w}, r\rangle,\langle\mathbf{v}, q\rangle)=B(\langle\mathbf{w}, r\rangle,\langle\mathbf{v}, q\rangle)-\varepsilon\left(\varphi_{h} r, q\right), \\
\langle\mathbf{w}, r\rangle,\langle\mathbf{v}, q\rangle \in \mathscr{V}_{b} .
\end{array}
$$

It follows from (2.3) and (2.12) that if $|\varepsilon| \leqslant c_{1} / 2$, then

$$
\sup _{\left\langle\mathbf{v}_{h}, q_{h}\right\rangle \in \mathscr{V}_{b h}} \frac{B_{\varepsilon}\left(\left\langle\mathbf{w}_{h}, r_{h}\right\rangle,\left\langle\mathbf{v}_{h}, q_{h}\right\rangle\right)}{\left\|\left\langle\mathbf{v}_{h}, q_{h}\right\rangle\right\|_{1,0}} \geqslant \frac{c_{1}}{2}\left\|\left\langle\mathbf{w}_{h}, r_{h}\right\rangle\right\|_{1,0}, \quad\left\langle\mathbf{w}_{h}, r_{h}\right\rangle \in \mathscr{V}_{b h} .
$$

Now if $\left\langle\mathbf{w}_{h}, r_{h}\right\rangle \in \mathscr{V}_{b h}$, we have from (2.2) and (2.11) that

$$
\begin{aligned}
& B_{\varepsilon}\left(\left\langle\mathbf{u}_{h \varepsilon}-\mathbf{w}_{h}, p_{h \varepsilon}-r_{h}\right\rangle,\left\langle\mathbf{v}_{h}, q_{h}\right\rangle\right) \\
& \quad=B_{\varepsilon}\left(\left\langle\mathbf{u}-\mathbf{w}_{h}, p-r_{h}\right\rangle,\left\langle\mathbf{v}_{h}, q_{h}\right\rangle\right)+\varepsilon\left(\varphi_{h} p, q_{h}\right), \quad\left\langle\mathbf{v}_{h}, q_{h}\right\rangle \in \mathscr{V}_{h h} .
\end{aligned}
$$

Thus, it follows from (3.2) that if $|\varepsilon| \leqslant c_{1} / 2$, then

$$
\frac{c_{1}}{2}\left\|\left\langle\mathbf{u}_{h \varepsilon}-\mathbf{w}_{h}, p_{h \varepsilon}-r_{h}\right\rangle\right\|_{1,0} \leqslant\left(c_{0}+\varepsilon\right)\left\|\left\langle\mathbf{u}-\mathbf{w}_{h}, p-r_{h}\right\rangle\right\|_{1,0}+|\varepsilon|\|p\| .
$$

Hence, we see that

$$
\begin{aligned}
\left\|\left\langle\mathbf{u}-\mathbf{u}_{h \varepsilon}, p-p_{h \varepsilon}\right\rangle\right\|_{1,0} \leqslant & \left(1+\frac{2\left(c_{0}+\varepsilon\right)}{c_{1}}\right) \inf _{\left\langle\mathbf{w}_{h}, r_{h}\right\rangle \in \mathscr{V}_{b h}}\left\|\left\langle\mathbf{u}-\mathbf{w}_{h}, p-r_{h}\right\rangle\right\|_{1,0} \\
& +\frac{2|\varepsilon|}{c_{1}}\|p\| .
\end{aligned}
$$

This proves Theorem 2 . We also note that since $\varphi_{h}$ was only required to satisfy the bound $\left|\varphi_{h}(x)\right| \leqslant 1, x \in \Omega$, in the above argument, this also proves Theorem 1 .

For $\xi \in L^{2}(\Omega)$, we define the norm

$$
\|\xi\|_{-1}=\sup _{\zeta \in H^{1}(\Omega)} \frac{(\xi, \zeta)}{\|\zeta\|_{1}}
$$

Now for $\langle\mathbf{v}, q\rangle \in L^{2}(\Omega)^{2} \times H^{1}(\Omega)$, let $\langle\mathbf{z}, m\rangle \in \mathscr{V}_{b}$ satisfy

$$
B(\langle\mathbf{w}, r\rangle,\langle\mathbf{z}, m\rangle)=(\mathbf{w}, \mathbf{v})+(r, q), \quad\langle\mathbf{w}, r\rangle \in \mathscr{V}_{b} .
$$

Then $\langle\mathbf{e}, E\rangle=\left\langle\mathbf{u}-\mathbf{u}_{h \varepsilon}, p-p_{h \varepsilon}\right\rangle$ satisfies

$$
\begin{aligned}
(\mathbf{e}, \mathbf{v}) & +(E, q)=B(\langle\mathbf{e}, E\rangle,\langle\mathbf{z}, m\rangle) \\
= & B\left(\langle\mathbf{e}, E\rangle,\left\langle\mathbf{z}-\mathbf{z}_{h}, m-m_{h}\right\rangle\right)+B\left(\langle\mathbf{e}, E\rangle,\left\langle\mathbf{z}_{h}, m_{h}\right\rangle\right) \\
= & B\left(\langle\mathbf{e}, E\rangle,\left\langle\mathbf{z}-\mathbf{z}_{h}, m-m_{h}\right\rangle\right)-\varepsilon\left(\varphi_{h} p_{h \varepsilon}, m_{h}\right), \quad\left\langle\mathbf{z}_{h}, m_{h}\right\rangle \in \mathscr{V}_{b h} .
\end{aligned}
$$

Now by (2.9) and (2.20), we can choose $\left\langle\mathbf{z}_{h}, m_{h}\right\rangle \in \mathscr{V}_{b h}$ so that

$$
\begin{aligned}
& B\left(\langle\mathbf{e}, E\rangle,\left\langle\mathbf{z}-\mathbf{z}_{h}, m-m_{h}\right\rangle\right) \\
& \quad \leqslant c_{0}\|\langle\mathbf{e}, E\rangle\|_{1,0}\left\|\left\langle\mathbf{z}-\mathbf{z}_{h}, m-m_{h}\right\rangle\right\|_{1,0} \\
& \leqslant c_{0}\|\langle\mathbf{e}, E\rangle\|_{1,0} c_{4} h\|\langle\mathbf{z}, m\rangle\|_{2,1} \leqslant c_{0} c_{3} c_{4} h\|\langle\mathbf{e}, E\rangle\|_{1,0}\|\langle\mathbf{v}, q\rangle\|_{0,1} .
\end{aligned}
$$


Also, we can assume that $\left\langle\mathbf{z}_{h}, m_{h}\right\rangle \in \mathscr{V}_{b h}$ is chosen so that

$$
\begin{aligned}
-\varepsilon\left(\varphi_{h} p_{h \varepsilon}, m_{h}\right) & =-\varepsilon\left(\varphi_{h} p, m_{h}\right)+\varepsilon\left(\varphi_{h}\left(p-p_{h \varepsilon}\right), m_{h}\right) \\
& =-\varepsilon\left(\varphi_{h} p, m\right)+\varepsilon\left(\varphi_{h} p, m-m_{h}\right)+\varepsilon\left(\varphi_{h}\left(p-p_{h \varepsilon}\right), m_{h}\right) \\
& \leqslant 2 c_{2}|\varepsilon| h\|p\|_{1}\|m\|_{1}+c_{4}|\varepsilon| h\|p\|\|m\|_{1}+c_{4}|\varepsilon|\|E\|\|m\|_{0} \\
& \leqslant\left(2 c_{2}|\varepsilon| h\|p\|_{1}+c_{4}|\varepsilon| h\|p\|+c_{4}|\varepsilon|\|E\|\right) c_{3}\|\langle\mathbf{v}, q\rangle\|_{0,1} .
\end{aligned}
$$

Hence, letting $\mathbf{v}=\mathbf{e}$ and $q=0$, we obtain from (3.7) and Theorem 2 that (with $\left.|\varepsilon| \leqslant c_{1} / 2\right)$

$$
\begin{aligned}
\|\mathbf{e}\| & \leqslant c_{0} c_{3} c_{4} h\|\langle\mathbf{e}, E\rangle\|_{1,0}+2 c_{2} c_{3}|\varepsilon| h\|p\|_{1}+c_{3} c_{4}|\varepsilon| h\|p\|+c_{3} c_{4}|\varepsilon|\|E\| \\
& \leqslant c\left(|\varepsilon| h\|\langle\mathbf{u}, p\rangle\|_{2,1}+|\varepsilon|^{2}\|p\|+h^{r+1}\|\langle\mathbf{u}, p\rangle\|_{r+1, r}\right) .
\end{aligned}
$$

This proves Theorem 3.

The following lemma also follows from the estimates (3.5), (3.6), and (3.7) by letting $\mathbf{v}=0$ and $q \in H^{1}(\Omega)$ be arbitrary.

Lemma 1. Let $|\varepsilon| \leqslant c_{1} / 2$. There exists a positive constant, $c$, such that if $\langle\mathbf{u}, p\rangle \in$ $H^{r+1}(\Omega)^{2} \times H^{r}(\Omega)$, then

$$
\left\|p-p_{h \varepsilon}\right\|_{-1} \leqslant c\left(|\varepsilon| h\|\langle\mathbf{u}, p\rangle\|_{2,1}+|\varepsilon|^{2}\|p\|+h^{r+1}\|\langle\mathbf{u}, p\rangle\|_{r+1, r}\right) .
$$

4. Estimates for the Smoothing Operator. In this section, we recall some estimates and relations for convolution with

$$
K(x)=K\left(x_{1}, x_{2}\right)= \begin{cases}(2 h)^{-2}, & \text { if }\left|x_{1}\right|,\left|x_{2}\right| \leqslant h, \\ 0 & \text { otherwise. }\end{cases}
$$

The proofs of these results can be found in [1]. We note that

$$
\int_{\mathbf{R}^{2}} K(x) d x=1
$$

We extend the notation of Section 2 by defining for $\mathscr{D} \subset \Omega$ and $k$ a positive integer,

$$
\begin{aligned}
& \|\xi\|_{\mathscr{D}}^{2}=\int_{\mathscr{D}} \xi^{2} d x, \quad \xi \in L^{2}(\mathscr{D}), \\
& \|\xi\|_{\mathscr{D}, k}^{2}=\sum_{|\alpha| \leqslant k}\left\|D^{\alpha} \xi\right\|_{\mathscr{D}}^{2}, \quad \xi \in H^{k}(\mathscr{D}), \\
& \|\xi\|_{\mathscr{D},-k}^{2}=\sup _{\eta \in H^{k}(\mathscr{D})} \frac{(\xi, \eta)}{\|\eta\|_{\mathscr{D}, k}}, \quad \xi \in L^{2}(\mathscr{D}), \\
& |\xi|_{\mathscr{D},-k}=\sup _{\eta \in H_{0}^{k}(\mathscr{D})} \frac{(\xi, \eta)}{\|\eta\|_{\mathscr{D}, k}}, \quad \xi \in L^{2}(\mathscr{D}),
\end{aligned}
$$

where

$$
H_{0}^{k}(\mathscr{D})=\text { closure of } C_{0}^{\infty}(\mathscr{D}) \text { in } H^{k}(\mathscr{D}) .
$$

We note that obviously

$$
|\xi|_{\mathscr{D},-k} \leqslant\|\xi\|_{\mathscr{D},-k}
$$


Now for $\operatorname{dist}(x, \partial \Omega)>\sqrt{2} h$, recall that $K * \xi(x)$ is well-defined and

$$
K * \xi(x)=(2 h)^{-2} \int_{x_{1}-h}^{x_{1}+h} \int_{x_{2}-h}^{x_{2}+h} \xi\left(y_{1}, y_{2}\right) d y_{1} d y_{2} .
$$

Now it is easy to check that if $\operatorname{dist}(x, \partial \Omega)>\sqrt{2} h$, then

$$
\begin{aligned}
\frac{\partial}{\partial x_{1}} K * \xi(x) & =(2 h)^{-1} \int_{x_{2}-h}^{x_{2}+h}\left[\frac{\xi\left(x_{1}+h, y_{2}\right)-\xi\left(x_{1}-h, y_{2}\right)}{2 h}\right] d y_{2} \\
& =L_{1} * \partial_{1, h} \xi(x)
\end{aligned}
$$

where

$$
L_{1}(x)= \begin{cases}\delta\left(x_{1}\right) / 2 h, & -h \leqslant x_{2} \leqslant h \\ 0 & \text { otherwise }\end{cases}
$$

and

$$
\partial_{1, h} \xi(x)=\frac{\xi\left(x_{1}+h, x_{2}\right)-\xi\left(x_{1}-h, x_{2}\right)}{2 h} .
$$

Here, $\delta\left(x_{1}\right)$ is the Dirac delta function. A similar definition can be given for $L_{2}(x)$ and $\partial_{2, h} \xi(x)$ so that

$$
\frac{\partial}{\partial x_{2}} K * \xi(x)=L_{2} * \partial_{2, h} \xi(x)
$$

We have the following lemmas [1].

LEMMA 2. If $\mathscr{D} \subset \subset \Omega$, then there exists a constant, $c_{5}$, such that for $h$ sufficiently small,

$$
\|\xi-K * \xi\|_{\mathscr{D}} \leqslant c_{5} h^{2}\|\xi\|_{2}, \quad \xi \in H^{2}(\Omega) .
$$

LEMMA 3. If $\mathscr{D}_{0} \subset \subset \mathscr{D} \subset \Omega$, then there exists a constant, $c_{6}$, such that

$$
\|\xi\|_{\mathscr{D}_{0}} \leqslant c_{6}\left(|\xi|_{\mathscr{D},-1}+\sum_{i=1}^{2}\left|\frac{\partial}{\partial x_{i}} \xi\right|_{\mathscr{D},-1}\right), \quad \xi \in L^{2}\left(\mathscr{D}_{1}\right) \text {. }
$$

LEMmA 4. If $\mathscr{D}_{0} \subset \subset \mathscr{D} \subset \Omega$, then there exists a constant, $c_{7}$, such that for $h$ sufficiently small and $\xi \in L^{2}\left(\mathscr{D}_{1}\right)$,

$$
\begin{aligned}
& \left|L_{i} * \xi\right|_{\mathscr{D}_{0},-1} \leqslant c_{7}|\xi|_{\mathscr{D},-1}, \quad i=1,2, \\
& |K * \xi|_{\mathscr{D}_{0},-1} \leqslant c_{7}|\xi|_{\mathscr{D},-1} .
\end{aligned}
$$

Now let $\mathscr{D}_{0} \subset \subset \mathscr{D}_{1} \subset \subset \mathscr{D}$ and suppose that $h$ is sufficiently small. Then we have that

$$
\begin{aligned}
\left\|p-K * p_{h \varepsilon}\right\|_{\mathscr{D}_{0}} & \leqslant\|p-K * p\|_{\mathscr{D}_{0}}+\left\|K *\left(p-p_{h_{\varepsilon}}\right)\right\|_{\mathscr{D}_{0}} \\
& \leqslant c_{5} h^{2}\|p\|_{2}+\left\|K *\left(p-p_{h \varepsilon}\right)\right\|_{\mathscr{D}_{0}}
\end{aligned}
$$

and from Lemma 3 and Lemma 4 we have that

$$
\begin{aligned}
\left\|K *\left(p-p_{h \varepsilon}\right)\right\|_{\mathscr{D}_{0}} & \leqslant c_{6}\left(\left|K *\left(p-p_{h_{\varepsilon}}\right)\right|_{\mathscr{D}_{1},-1}+\sum_{i=1}^{2}\left|\frac{\partial}{\partial x_{i}} K *\left(p-p_{h_{\varepsilon}}\right)\right|_{\mathscr{D}_{1},-1}\right) \\
& =c_{6}\left(\left|K *\left(p-p_{h_{\varepsilon}}\right)\right|_{\mathscr{D}_{1},-1}+\sum_{i=1}^{2}\left|L_{i} * \partial_{i, h}\left(p-p_{h_{\varepsilon}}\right)\right|_{\mathscr{D}_{1},-1}\right) \\
& \leqslant c_{6} c_{7}\left(\left.\left|p-p_{\left.h_{\varepsilon}\right|_{\mathscr{D},-1}}+\sum_{i=1}^{2}\right| \partial_{i, h}\left(p-p_{h \varepsilon}\right)\right|_{\mathscr{D},-1}\right) .
\end{aligned}
$$


Thus

$$
\begin{aligned}
\left\|p-K * p_{h \varepsilon}\right\|_{\mathscr{D}_{0}} \leqslant & c_{5} h^{2}\|p\|_{2}+c_{6} c_{7}\left|p-p_{h \varepsilon}\right|_{\mathscr{D},-1} \\
& +c_{6} c_{7} \sum_{i=1}^{2}\left|\partial_{i, h}\left(p-p_{h \varepsilon}\right)\right|_{\mathscr{D},-1} .
\end{aligned}
$$

Now, by Lemma 1,

$$
\begin{aligned}
\left|p-p_{h \varepsilon}\right|_{\mathscr{D},-1} & \leqslant\left\|p-p_{h \varepsilon}\right\|_{-1} \\
& \leqslant c\left(|\varepsilon| h\|\langle\mathbf{u}, p\rangle\|_{2,1}+|\varepsilon|^{2}\|p\|+h^{r+1}\|\langle\mathbf{u}, p\rangle\|_{r+1, r}\right) .
\end{aligned}
$$

The next section will be devoted to estimating

$$
\left\|\partial_{i, h}\left(p-p_{h \varepsilon}\right)\right\|_{\mathscr{D},-1} \text {. }
$$

5. Estimates for Difference Quotients. The results in this section are motivated by the interior estimates for difference quotients for Ritz-Galerkin methods developed in [8]. We assume that there exists $\tilde{\Omega} \subset \subset \Omega$ with the property that for $h$ sufficiently small,

$$
\partial_{i, h} \varphi_{h}=0, \quad i=1,2,
$$

where

$$
\partial_{i, h} \xi(x)=\frac{\xi\left(x+h e_{i}\right)-\xi\left(x-h e_{i}\right)}{2 h}, \quad x \in \tilde{\Omega},
$$

and $e_{1}=(1,0), e_{2}=(0,1)$. We note that if $\left|\varphi_{h}(x)\right| \equiv 1$ and $\varphi_{h}$ alternates sign on the rows of a rectangular triangulation (see Figure 2) in a neighborhood of $\tilde{\Omega}$, then (5.1) is satisfied.

We define for arbitrary open sets $\mathscr{D} \subset \subset \Omega$, the spaces

$$
\mathscr{V}(\mathscr{D})=\left\{\langle\mathbf{v}, q\rangle \in \mathscr{V}_{b} \mid \operatorname{supp}\left\langle\mathbf{v}_{h}, q\right\rangle \subseteq \mathscr{D}\right\}
$$

and

$$
\mathscr{V}_{h}(\mathscr{D})=\left\{\left\langle\mathbf{v}_{h}, q_{h}\right\rangle \in \mathscr{V}_{\text {bh }} \mid \operatorname{supp}\left\langle\mathbf{v}_{h}, q_{h}\right\rangle \subseteq \mathscr{D}\right\},
$$

and we shall also assume that $\tilde{\Omega} \subset \subset \Omega$ has the property that for $h$ sufficiently small,

$$
\partial_{i, h}\left(\left\langle\mathbf{v}_{h}, q_{h}\right\rangle\right) \in \mathscr{V}_{b h} \text { for }\left\langle\mathbf{v}_{h}, q_{h}\right\rangle \in \mathscr{V}_{h}(\tilde{\Omega}) .
$$

We note that (5.2) is valid for the usual finite element spaces of piecewise polynomials with respect to a triangulation which is regular in a neighborhood of $\tilde{\Omega}$ [8].

It then follows from (5.1) and (5.2) that for $\mathbf{e}=\mathbf{u}-\mathbf{u}_{h \varepsilon}$ and $E=p-p_{h \varepsilon}$ we have (assuming $h$ is sufficiently small)

$$
\begin{aligned}
& B\left(\left\langle\partial_{i, h} \mathbf{e}, \partial_{i, h} E\right\rangle,\left\langle\mathbf{v}_{h}, q_{h}\right\rangle\right) \\
& \quad=-B\left(\langle\mathbf{e}, E\rangle,\left\langle\partial_{i, h} \mathbf{v}_{h}, \partial_{i, h} q_{h}\right\rangle\right)=\varepsilon\left(\varphi_{h} p_{h \varepsilon}, \partial_{i, h} q_{h}\right)=-\varepsilon\left(\partial_{i, h}\left(\varphi_{h} p_{h \varepsilon}\right), q_{h}\right) \\
& \quad=-\varepsilon\left(\varphi_{h}\left(x+h e_{i}\right) \partial_{i, h} p_{h \varepsilon}, q_{h}\right), \quad\left\langle\mathbf{v}_{h}, q_{h}\right\rangle \in \mathscr{V}_{h}(\tilde{\Omega}) .
\end{aligned}
$$

We also need to assume that for arbitrary open sets $\mathscr{D}_{1} \subset \subset \mathscr{D} \subset \subset \Omega$ there exists a constant $c_{8}>0, c_{8}=c_{8}\left(\mathscr{D}_{1}, \mathscr{D}\right)$, such that for $h$ sufficiently small and $\langle\mathbf{v}, q\rangle \in$ $\mathscr{V}\left(\mathscr{D}_{1}\right) \cap H^{r+1}(\Omega)^{2} \times H^{r}(\Omega)$ there exists $\left\langle\mathbf{v}_{h}, q_{h}\right\rangle \in \mathscr{V}_{h}(\mathscr{D})$ with the property that

$$
\left\|\left\langle\mathbf{v}-\mathbf{v}_{h}, q-q_{h}\right\rangle\right\|_{1,0} \leqslant c_{8} h^{s}\|\langle\mathbf{v}, q\rangle\|_{s+1, s}, \quad 0 \leqslant s \leqslant r .
$$


We further assume that if $\omega \in C_{0}^{\infty}\left(\mathscr{D}_{1}\right)$, then there exists a constant $c_{9}>0$, $c_{9}=c_{9}\left(\mathscr{D}_{1}, \mathscr{D}, \omega\right)$, such that for $h$ sufficiently small and $\left\langle\mathbf{w}_{h}, r_{h}\right\rangle \in \mathscr{V}_{h}(\mathscr{D})$, there exists $\left\langle\mathbf{v}_{h}, q_{h}\right\rangle \in \mathscr{V}_{h}(\mathscr{D})$ such that

$$
\left\|\left\langle\omega \mathbf{w}_{h}-\mathbf{v}_{h}, \omega r_{h}-q_{h}\right\rangle\right\|_{1,0} \leqslant c_{9} h\left\|\left\langle\mathbf{w}_{h}, r_{h}\right\rangle\right\|_{1,0} .
$$

We note that these approximability assumptions hold for the spaces $M_{h}$ and $X_{h}$ discussed in Section 2, as well as for other stable finite element spaces used to approximate viscous, incompressible flow.

If $\mathscr{D}$ is an annulus, let its inner boundary be denoted by $\partial \mathscr{D}_{\text {in }}$ and let its outer boundary be denoted by $\partial \mathscr{D}_{\text {out }}$. We define the spaces

$$
\begin{aligned}
& H_{b}^{1}(\mathscr{D})=\left\{\mathbf{v} \in H^{1}(\mathscr{D})^{2} \mid \mathbf{v}=0 \text { on } \partial_{\text {out }}\right\}, \\
& \mathscr{V}_{b}(\mathscr{D})=\left\{\langle\mathbf{v}, \mathbf{q}\rangle \mid \mathbf{v} \in H_{b}^{1}(\mathscr{D}) \text { and } q \in L^{2}(\mathscr{D})\right\} .
\end{aligned}
$$

Henceforth, open sets $\mathscr{D} \subset \subset \Omega$ will be annuli. For $\mathbf{v}: \mathscr{D} \rightarrow \mathbf{R}^{2}, \xi: \mathscr{D} \rightarrow \mathbf{R}$, and $l, m \geqslant 0$ integers, we can define the norm

$$
\|\langle\mathbf{v}, \xi\rangle\|_{\mathscr{D}, l, m}^{2}=\|\mathbf{v}\|_{\mathscr{D}, l}^{2}=\|\xi\|_{\mathscr{D}, m}^{2}, \quad \mathbf{v} \in H^{l}(\mathscr{D})^{2}, \xi \in H^{m}(\mathscr{D}) .
$$

We also extend the definition of negative norms to vector-valued functions and we define

$$
\begin{aligned}
\|\langle\mathbf{w}, r\rangle\|_{\mathscr{D},-l,-m}^{2} & =\|\mathbf{w}\|_{\mathscr{D},-l}^{2}+\|r\|_{\mathscr{D},-m}^{2}, \\
|\langle\mathbf{w}, r\rangle|_{\mathscr{D},-l,-m}^{2} & =|\mathbf{w}|_{\mathscr{D},-l}^{2}+|r|_{\mathscr{D},-m}^{2} .
\end{aligned}
$$

We can define $B(\langle\mathbf{w}, r\rangle,\langle\mathbf{v}, q\rangle)$ for $\langle\mathbf{w}, r\rangle,\langle\mathbf{v}, q\rangle \in \mathscr{V}_{b}(\mathscr{D})$ as in Section 2 with $\Omega$ replaced by $\mathscr{D}$. As before, it is known that there exists a positive constant, $c_{1}=c_{1}(\mathscr{D})$, such that [3], [9]

$$
\sup _{\langle\mathbf{v}, q\rangle \in \mathscr{V}_{b}(\mathscr{D})} \frac{B(\langle\mathbf{w}, r\rangle,\langle\mathbf{v}, q\rangle)}{\|\langle\mathbf{v}, q\rangle\|_{\mathscr{D}, 1,0}} \geqslant c_{1}\|\langle\mathbf{w}, r\rangle\|_{\mathscr{D}, 1,0}, \quad\langle\mathbf{w}, r\rangle \in \mathscr{V}_{b}(\mathscr{D}),
$$

and a positive constant, $c_{0}=c_{0}(\mathscr{D})$, such that

$$
B(\langle\mathbf{w}, r\rangle,\langle\mathbf{v}, q\rangle) \leqslant c_{0}\|\langle\mathbf{w}, r\rangle\|_{\mathscr{D}, 1,0}\|\langle\mathbf{v}, q\rangle\|_{\mathscr{D}, 1,0}, \quad\langle\mathbf{w}, r\rangle,\langle\mathbf{v}, q\rangle \in \mathscr{V}_{b}(\mathscr{D}) .
$$

It is also known that the regularity result (2.19) holds on $\mathscr{D}$ with $c_{3}=c_{3}(\mathscr{D})$.

Finally, we will set

$$
\mathscr{V}_{b h}(\mathscr{D})=\left\{\left\langle\mathbf{w}_{h}, r_{h}\right\rangle \in \mathscr{V}_{b h} \mid \mathbf{w}_{h} \in H_{b}^{1}(\mathscr{D}) \text { and supp } r_{h} \subset \mathscr{D}\right\}
$$

and suppose that the stability result $(2.3)$ holds for $\mathscr{V}_{h h}(\mathscr{D})$ with $c_{1}=c_{1}(\mathscr{D})>0$, i.e., for $h$ sufficiently small,

$$
\begin{aligned}
\sup _{\left\langle\mathbf{v}_{h}, q_{h}\right\rangle \in \mathscr{V}_{h h}(\mathscr{D})} \frac{B\left(\left\langle\mathbf{w}_{h}, r_{h}\right\rangle,\left\langle\mathbf{v}_{h}, q_{h}\right\rangle\right)}{\left\|\left\langle\mathbf{v}_{h}, q_{h}\right\rangle\right\|_{\mathscr{D}, 1,0}} \geqslant c_{1}\left\|\left\langle\mathbf{w}_{h}, r_{h}\right\rangle\right\|_{\mathscr{D}, 1,0}, \\
\left\langle\mathbf{w}_{h}, r_{h}\right\rangle \in \mathscr{V}_{b h}(\mathscr{D}) .
\end{aligned}
$$

We note that there exist families of triangulations, $\left\{\mathscr{T}_{h}\right\}$, for which the properties of the triangulation with respect to the annular domain, $\Omega$, described in Section 2 hold for $\mathscr{D}$. In that case, the argument to prove (5.6) for the constant space, $\mathscr{M}_{h}$, and the quadratic space, $\chi_{h}$, is identical to the argument given to prove (2.3). We will prove the following result for the approximation of the pressure by the variable penalty method under the above hypotheses. 
THEOREM 4. Let $|\varepsilon| \leqslant c_{1} / 2$ and let $\Omega_{1} \subset \subset \tilde{\Omega}$. There exists a positive constant, $c$, independent of $h$ and $\varepsilon$, so that if $\langle\mathbf{u}, p\rangle \in H^{r+2}(\Omega)^{2} \times H^{r+1}(\Omega)$ and $h$ is sufficiently small, then

$$
\begin{aligned}
& \left\|p-K * p_{h, \varepsilon}\right\|_{\Omega_{1}} \\
& \quad \leqslant c\left(|\varepsilon| h\|\langle\mathbf{u}, p\rangle\|_{2,2}+|\varepsilon|^{2}\|p\|_{1}+h^{2}\|p\|_{2}+h^{r+1}\|\langle\mathbf{u}, p\rangle\|_{r+2, r+1}\right) .
\end{aligned}
$$

Now any $\Omega_{1} \subset \subset \tilde{\Omega} \subset \subset \Omega$ can be covered by a finite number of annuli, each of which is compact in $\tilde{\Omega}$. Thus, for the proof of Theorem 4 we may assume that $\Omega_{1}$ is an annulus. We will prove Theorem 4 in a sequence of lemmas.

LeMma 5. Let $\mathscr{D}_{0}$ and $\mathscr{D}$ be concentric annuli, $\mathscr{D}_{0} \subset \subset \mathscr{D} \subset \subset \tilde{\Omega}$. Then, for $h$ sufficiently small, there exists a positive constant, $c$, such that

$$
\begin{aligned}
& \left\|\left\langle\partial_{i, h} \mathbf{e}, \partial_{i, h} E\right\rangle\right\|_{\mathscr{D}_{0}, 0,-1} \\
& \begin{aligned}
\leqslant c\left(h\left\|\left\langle\partial_{i, h} \mathbf{e}, \partial_{i, h} E\right\rangle\right\|_{\mathscr{D}, 1,0}+\left|\left\langle\partial_{i, h} \mathbf{e}, \partial_{i, h} E\right\rangle\right|_{\mathscr{D},-1,-2}\right. & \\
& \left.+|\varepsilon| h\|\langle\mathbf{u}, p\rangle\|_{2,2}+|\varepsilon|^{2}\|p\|\right) .
\end{aligned}
\end{aligned}
$$

Proof of Lemma 5. Let $\mathscr{D}^{\prime}$ be an annulus such that $\mathscr{D}_{0} \subset \subset \mathscr{D}^{\prime} \subset \subset \mathscr{D}$ are concentric annuli and let $\omega \in C_{0}^{\infty}\left(\mathscr{D}^{\prime}\right)$ with $\omega \equiv 1$ on $\mathscr{D}_{0}$. Then, we have

$$
\begin{aligned}
\left\|\left\langle\partial_{i, h} \mathbf{e}, \partial_{i, h} E\right\rangle\right\|_{\mathscr{D}_{0}, 0,-1} & \leqslant\left\|\left\langle\omega \partial_{i, h} \mathbf{e}, \omega \partial_{i, h} E\right\rangle\right\|_{\mathscr{D}, 0,-1} \\
& =\sup _{\langle\mathbf{v}, q\rangle \in L^{2}(\mathscr{D})^{2} \times H^{1}(\mathscr{D})} \frac{\left(\omega \partial_{i, h} \mathbf{e}, \mathbf{v}\right)+\left(\omega \partial_{i, h} E, q\right)}{\|\langle\mathbf{v}, q\rangle\|_{\mathscr{D}, 0,1}} .
\end{aligned}
$$

Now let $\langle\mathbf{z}, m\rangle \in \mathscr{V}_{b}(\mathscr{D})$ be the solution to

$$
B(\langle\mathbf{w}, r\rangle,\langle\mathbf{z}, m\rangle)=(\mathbf{w}, \mathbf{v})+(r, q), \quad\langle\mathbf{w}, r\rangle \in \mathscr{V}_{b}(\mathscr{D})
$$

where $\langle\mathbf{v}, q\rangle \in L^{2}(\mathscr{D})^{2} \times H^{1}(\mathscr{D})$. Then,

$$
\|\langle\mathbf{z}, m\rangle\|_{\mathscr{D}, 2,1} \leqslant c_{3}\|\langle\mathbf{v}, q\rangle\|_{\mathscr{D}, 0,1} .
$$

Hence, for $\langle\mathbf{v}, q\rangle \in L^{2}(\mathscr{D})^{2} \times H^{1}(\mathscr{D})$,

$$
\begin{aligned}
& \left(\omega \partial_{i, h} \mathbf{e}, \mathbf{v}\right)+\left(\omega \partial_{i, h} E, q\right)=B\left(\left\langle\omega \partial_{i, h} \mathbf{e}, \omega \partial_{i, h} E\right\rangle,\langle\mathbf{z}, m\rangle\right) \\
& \quad=B\left(\left\langle\partial_{i, h} \mathbf{e}, \partial_{i, h} E\right\rangle,\langle\omega \mathbf{z}, \omega m\rangle\right)+I\left(\left\langle\partial_{i, h} \mathbf{e}, \partial_{i, h} E\right\rangle,\langle\mathbf{z}, m\rangle\right)
\end{aligned}
$$

where

$$
\begin{aligned}
& I\left(\left\langle\partial_{i, h} \mathbf{e}, \partial_{i, h} E\right\rangle,\langle\mathbf{z}, m\rangle\right) \\
& =2\left(\frac{\partial \omega}{\partial x_{k}} \partial_{i, h} e_{j}, D_{j k}(\mathbf{z})\right)+2\left(\partial_{i, h} e_{j}, \frac{\partial}{\partial x_{k}}\left(\frac{\partial \omega}{\partial x_{j}} z_{k}+\frac{\partial \omega}{\partial x_{k}} z_{j}\right)\right) \\
& \quad+\left(\left(\partial_{i, h} E\right) \nabla \omega, \mathbf{z}\right)+\left(\partial_{i, h} \mathbf{e},(\nabla \omega) m\right) \\
& \leqslant c\left|\left\langle\partial_{i, h} \mathbf{e}, \partial_{i, h} E\right\rangle\right|_{\mathscr{D},-1,-2}\|\langle\mathbf{z}, m\rangle\|_{\mathscr{D}, 2,1} \\
& \leqslant c c_{3}\left|\left\langle\partial_{i, h} \mathbf{e}, \partial_{i, h} E\right\rangle\right|_{\mathscr{D},-1,-2}\|\langle\mathbf{v}, q\rangle\|_{\mathscr{D}, 0,1} .
\end{aligned}
$$

Now, by (5.3) for arbitrary $\left\langle\mathbf{z}_{h}, m_{h}\right\rangle \in \mathscr{V}_{h}(\mathscr{D})$,

$$
\begin{aligned}
& B\left(\left\langle\partial_{i, h} \mathbf{e}, \partial_{i, h} E\right\rangle,\langle\omega \mathbf{z}, \omega m\rangle\right) \\
& \quad=B\left(\left\langle\partial_{i, h} \mathbf{e}, \partial_{i, h} E\right\rangle,\left\langle\omega \mathbf{z}-\mathbf{z}_{h}, \omega m-m_{h}\right\rangle\right)-\varepsilon\left(\varphi_{h} \partial_{i, h} p_{h \varepsilon}, m_{h}\right) .
\end{aligned}
$$

Now, by (5.4), we can choose $\left\langle\mathbf{z}_{h}, m_{h}\right\rangle \in \mathscr{V}_{h}(\mathscr{D})$, so that

$$
\left\|\left\langle\omega \mathbf{z}-\mathbf{z}_{h}, \omega m-m_{h}\right\rangle\right\|_{1,0} \leqslant c_{8} h\|\langle\omega \mathbf{z}, \omega m\rangle\|_{\mathscr{D}, 2,1} .
$$


Hence,

$$
\begin{aligned}
& B\left(\left\langle\partial_{i, h} \mathbf{e}, \partial_{i, h} E\right\rangle,\left\langle\omega \mathbf{z}-\mathbf{z}_{h}, \omega m-m_{h}\right\rangle\right) \\
& \quad \leqslant c_{0}\left\|\left\langle\partial_{i, h} \mathbf{e}, \partial_{i, h} E\right\rangle\right\|_{\mathscr{D}, 1,0} c_{8} h\|\langle\omega \mathbf{z}, \omega m\rangle\|_{\mathscr{D}, 2,1} \\
& \quad \leqslant c h\left\|\left\langle\partial_{i, h} \mathbf{e}, \partial_{i, h} E\right\rangle\right\|_{\mathscr{D}, 1,0}\|\langle\mathbf{v}, q\rangle\|_{\mathscr{D}, 0,1}
\end{aligned}
$$

Further,

$$
\begin{aligned}
-\left(\varphi_{h} \partial_{i, h} p_{h \varepsilon}, m_{h}\right)= & \left(\varphi_{h} \partial_{i, h} p_{h \varepsilon}, \omega m-m_{h}\right)-\left(\varphi_{h} \partial_{i, h} p_{h \varepsilon}, \omega m\right) \\
= & \left(\varphi_{h} \partial_{i, h} p, \omega m-m_{h}\right)+\left(\varphi_{h} \partial_{i, h}\left(p_{h \varepsilon}-p\right), \omega m-m_{h}\right) \\
& -\left(\varphi_{h} \partial_{i, h} p, \omega m\right)+\left(\varphi_{h} \partial_{i, h}\left(p-p_{h \varepsilon}\right), \omega m\right) .
\end{aligned}
$$

Now, by (5.15),

$$
\begin{array}{r}
\left(\varphi_{h} \partial_{i, h} p, \omega m-m_{h}\right) \leqslant c\|p\|_{1}\left\|\omega m-m_{h}\right\| \leqslant c h\|p\|_{1}\|\langle\omega \mathbf{z}, \omega m\rangle\|_{\mathscr{D}, 2,1}, \\
\left(\varphi_{h} \partial_{i, h}\left(p_{h \varepsilon}-p\right), \omega m-m_{h}\right) \leqslant \frac{\|E\|}{h}\left\|\omega m-m_{h}\right\| \leqslant c\|E\|\|\langle\omega \mathbf{z}, \omega m\rangle\|_{\mathscr{D}, 2,1},
\end{array}
$$

by $(2.13)$,

$$
-\left(\varphi_{h} \partial_{i, h} p, \omega m\right) \leqslant c h\|p\|_{2}\|\omega m\|_{\mathscr{D}, 1}
$$

and

$$
\left(\varphi_{h} \partial_{i, h}\left(p-p_{h \varepsilon}\right), \omega m\right)=-\left(\varphi_{h}\left(x+h e_{i}\right) E, \partial_{i, h} \omega m\right) \leqslant c\|E\|\|\omega m\|_{\mathscr{D}, 1}
$$

Thus,

$$
\begin{aligned}
-\left(\varphi_{h} \partial_{i, h} p_{h \varepsilon}, m_{h}\right) & \leqslant c\left(h\|p\|_{2}+\|E\|\right)\|\langle\mathbf{z}, m\rangle\|_{\mathscr{D}, 2,1} \\
& \leqslant c\left(h\|p\|_{2}+\|E\|\right)\|\langle\mathbf{v}, q\rangle\|_{\mathscr{D}, 0,1}
\end{aligned}
$$

The result of Lemma 5 now follows from (2.20) and Theorem 2.

Now, for $h$ sufficiently small, by Theorem 3 and Lemma 1 ,

$$
\begin{aligned}
& \left|\left\langle\partial_{i, h} \mathbf{e}, \partial_{i, h} E\right\rangle\right|_{\mathscr{D},-1,-2} \leqslant c\|\langle\mathbf{e}, E\rangle\|_{0,-1} \\
& \quad \leqslant c|\varepsilon| h\|\langle\mathbf{u}, p\rangle\|_{2,1}+c|\varepsilon|^{2}\|p\|+c h^{r+1}\|\langle\mathbf{u}, p\rangle\|_{r+1, r} .
\end{aligned}
$$

Hence, we have from Lemma 5, that

$$
\begin{aligned}
\left\|\left\langle\partial_{i, h} \mathbf{e}, \partial_{i, h} E\right\rangle\right\|_{\mathscr{D}_{0}, 0,-1} & \\
\leqslant c\left(h\left\|\left\langle\partial_{i, h} \mathbf{e}, \partial_{i, h} E\right\rangle\right\|_{\mathscr{D}, 1,0}+|\varepsilon| h\|\langle\mathbf{u}, p\rangle\|_{2,2}\right. & \\
& \left.+|\varepsilon|^{2}\|p\|+h^{r+1}\|\langle\mathbf{u}, p\rangle\|_{r+1, r}\right) .
\end{aligned}
$$

Thus, it remains to estimate $h\left\|\left\langle\partial_{i, h} \mathbf{e}, \partial_{i, h} E\right\rangle\right\|_{\mathscr{D}, 1,0}$.

We first prove the following lemma.

LEMMA 6. Let $\mathscr{D}_{0} \subset \subset \mathscr{D}$ be concentric annuli. Then there exists a positive constant, $c$, such that for $h$ sufficiently small and $\left\langle\mathbf{w}_{h}, r_{h}\right\rangle \in \mathscr{V}_{h}(\mathscr{D})$ satisfying

we have

$$
B\left(\left\langle\mathbf{w}_{h}, r_{h}\right\rangle,\left\langle\mathbf{v}_{h}, q_{h}\right\rangle\right)=0, \quad\left\langle\mathbf{v}_{h}, q_{h}\right\rangle \in \mathscr{V}_{h}(\mathscr{D}),
$$

$$
\left\|\left\langle\mathbf{w}_{h}, r_{h}\right\rangle\right\|_{\mathscr{D}_{0}, 1,0} \leqslant c\left(h\left\|\left\langle\mathbf{w}_{h}, r_{h}\right\rangle\right\|_{\mathscr{D}, 1,0}+\left|\left\langle\mathbf{w}_{h}, r_{h}\right\rangle\right|_{\mathscr{D}, 0,-1}\right) .
$$


Proof. Let $\mathscr{D}^{\prime}$ be an annulus such that $\mathscr{D}_{0} \subset \subset \mathscr{D}^{\prime} \subset \subset \mathscr{D}$ are concentric annuli, and let $\omega \in C_{0}^{\infty}\left(\mathscr{D}^{\prime}\right)$ with $\omega \equiv 1$ on $\mathscr{D}_{0}$. Let $\left\langle\mathbf{W}_{h}, R_{h}\right\rangle \in \mathscr{V}_{b h}(\mathscr{D})$ satisfy

$$
B\left(\left\langle\omega \mathbf{w}_{h}-\mathbf{w}_{h}, \omega r_{h}-R_{h}\right\rangle,\left\langle\mathbf{v}_{h}, q_{h}\right\rangle\right)=0, \quad\left\langle\mathbf{v}_{h}, q_{h}\right\rangle \in \mathscr{V}_{b h}(\mathscr{D}) .
$$

Then by (5.5) and (5.6),

$$
\begin{aligned}
\left\|\left\langle\omega \mathbf{w}_{h}-\mathbf{w}_{h}, \omega r_{h}-R_{h}\right\rangle\right\|_{\mathscr{D}, 1,0} \\
\quad \leqslant\left(1+\frac{c_{0}}{c_{1}}\right) \inf _{\left\langle\mathbf{v}_{h}, q_{h}\right\rangle \in \mathscr{V}_{h h}(\mathscr{D})}\left\|\left\langle\omega \mathbf{w}_{h}-\mathbf{v}_{h}, \omega r_{h}-q_{h}\right\rangle\right\|_{\mathscr{D}, 1,0} \\
\leqslant \operatorname{ch}\left\|\left\langle\mathbf{w}_{h}, r_{h}\right\rangle\right\|_{\mathscr{D}, 1,0} .
\end{aligned}
$$

Thus,

$$
\begin{aligned}
\left\|\left\langle\mathbf{w}_{h}, r_{h}\right\rangle\right\|_{\mathscr{D}_{0}, 1,0} & \leqslant\left\|\left\langle\omega \mathbf{w}_{h}, \omega r_{h}\right\rangle\right\|_{\mathscr{D}, 1,0} \\
& \leqslant\left\|\left\langle\omega \mathbf{w}_{h}-\mathbf{W}_{h}, \omega r_{h}-R_{h}\right\rangle\right\|_{\mathscr{D}, 1,0}+\left\|\left\langle\mathbf{W}_{h}, R_{h}\right\rangle\right\|_{\mathscr{D}, 1,0} \\
& \leqslant c h\left\|\left\langle\mathbf{w}_{h}, r_{h}\right\rangle\right\|_{\mathscr{D}, 1,0}+\left\|\left\langle\mathbf{W}_{h}, R_{h}\right\rangle\right\|_{\mathscr{D}, 1,0}
\end{aligned}
$$

Now, by (5.6),

$$
\left\|\left\langle\mathbf{W}_{h}, R_{h}\right\rangle\right\|_{\mathscr{D}, 1,0} \leqslant c_{1}^{-1} \sup _{\left\langle\mathbf{v}_{h}, q_{h}\right\rangle \in \mathscr{V}_{h h}(\mathscr{D})} \frac{B\left(\left\langle\mathbf{W}_{h}, R_{h}\right\rangle,\left\langle\mathbf{v}_{h}, q_{h}\right\rangle\right)}{\left\|\left\langle\mathbf{v}_{h}, q_{h}\right\rangle\right\|_{\mathscr{D}, 1,0}},
$$

and for $\left\langle\mathbf{v}_{h}, q_{h}\right\rangle \in \mathscr{V}_{b h}(\mathscr{D})$,

$$
\begin{aligned}
& B\left(\left\langle\mathbf{w}_{h}, R_{h}\right\rangle,\left\langle\mathbf{v}_{h}, q_{h}\right\rangle\right)=B\left(\left\langle\omega \mathbf{w}_{h}, \omega r_{h}\right\rangle,\left\langle\mathbf{v}_{h}, q_{h}\right\rangle\right) \\
& \quad=B\left(\left\langle\mathbf{w}_{h}, r_{h}\right\rangle,\left\langle\omega \mathbf{v}_{h}, \omega q_{h}\right\rangle\right)+I\left(\left\langle\mathbf{w}_{h}, r_{h}\right\rangle,\left\langle\mathbf{v}_{h}, q_{h}\right\rangle\right),
\end{aligned}
$$

where

$$
\begin{aligned}
I\left(\left\langle\mathbf{w}_{h}, r_{h}\right\rangle,\left\langle\mathbf{v}_{h}, q_{h}\right\rangle\right)= & 2\left(\frac{\partial \omega}{\partial x_{k}} w_{h j}, D_{j k}\left(\mathbf{v}_{h}\right)\right) \\
& +2\left(w_{h j}, \frac{\partial}{\partial x_{k}}\left(\frac{\partial \omega}{\partial x_{j}} v_{h k}+\frac{\partial \omega}{\partial x_{k}}, v_{h j}\right)\right) \\
& +\left(r_{h} \nabla \omega, \mathbf{v}_{h}\right)+\left(\mathbf{w}_{h}, \nabla \omega q_{h}\right) \\
\leqslant & c\left|\left\langle\mathbf{w}_{h}, r_{h}\right\rangle\right|_{\mathscr{D}, 0,-1}\left\|\left\langle\mathbf{v}_{h}, q_{h}\right\rangle\right\|_{\mathscr{D}, 1,0}
\end{aligned}
$$

and by (5.5) we have for $\left\langle\mathbf{V}_{h}, Q_{h}\right\rangle \in \mathscr{V}_{h}(\mathscr{D}) \subset \mathscr{V}_{b h}(\mathscr{D})$,

$$
\begin{aligned}
B\left(\left\langle\mathbf{w}_{h}, r_{h}\right\rangle,\left\langle\omega \mathbf{v}_{h}, \omega q_{h}\right\rangle\right) & =B\left(\left\langle\mathbf{w}_{h}, r_{h}\right\rangle,\left\langle\omega \mathbf{v}_{\mathbf{h}}-\mathbf{v}_{\mathbf{h}}, \omega q_{h}-Q_{h}\right\rangle\right) \\
& \leqslant c\left\|\left\langle\mathbf{w}_{\mathbf{h}}, r_{h}\right\rangle\right\|_{\mathscr{D}, 1,0} \operatorname{ch}\left\|\left\langle\mathbf{v}_{\mathbf{h}}, q_{h}\right\rangle\right\|_{\mathscr{D}, 1,0} \\
& \leqslant c h\left\|\left\langle\mathbf{w}_{\mathbf{h}}, r_{h}\right\rangle\right\|_{\mathscr{D}, 1,0}\left\|\left\langle\mathbf{v}_{\mathbf{h}}, q_{h}\right\rangle\right\|_{\mathscr{D}, 1,0} .
\end{aligned}
$$

Thus, it follows from (5.22)-(5.24) that

$$
\left\|\left\langle\mathbf{W}_{h}, R_{h}\right\rangle\right\|_{\mathscr{D}, 1,0} \leqslant c\left(h\left\|\left\langle\mathbf{w}_{h}, r_{h}\right\rangle\right\|_{\mathscr{D}, 1,0}+\left|\left\langle\mathbf{w}_{h}, r_{h}\right\rangle\right|_{\mathscr{D}, 0,-1}\right) .
$$

The result of Lemma 6 follows from (5.21) and (5.25).

Finally, we prove the following lemma.

LEMMA 7. Let $\mathscr{D}_{0}$ be an annulus. Then there exists a constant, $c>0$, such that for $h$ sufficiently small,

(5.26) $h\left\|\left\langle\partial_{i, h} \mathbf{e}, \partial_{i, h} E\right\rangle\right\|_{\mathscr{D}_{0}, 1,0} \leqslant c\left(|\varepsilon| h\|\langle\mathbf{u}, p\rangle\|_{2,1}+|\varepsilon|^{2}\|p\|_{1}+h^{r+1}\|\langle\mathbf{u}, p\rangle\|_{r+2, r+1}\right)$. 
Proof. Let $\mathscr{D}_{0}^{\prime}, \mathscr{D}^{\prime}$, and $\mathscr{D}$ be annuli such that $\mathscr{D}_{0} \subset \subset \mathscr{D}_{0}^{\prime} \subset \subset \mathscr{D}^{\prime} \subset \subset \mathscr{D} \subset \subset \tilde{\Omega}$ are concentric annuli, and let $\omega \in C_{0}^{\infty}\left(\mathscr{D}^{\prime}\right)$ with $\omega \equiv 1$ on $\mathscr{D}_{0}^{\prime}$. Next, let $\left\langle\mathbf{W}_{h}, R_{h}\right\rangle \in$ $\mathscr{V}_{b h}(\mathscr{D})$ be the solution to

$$
\begin{aligned}
B\left(\left\langle\mathbf{W}_{h}, R_{h}\right\rangle,\left\langle\mathbf{v}_{h}, q_{h}\right\rangle\right)= & B\left(\left\langle\omega \partial_{i, h} \mathbf{u}, \omega \partial_{i, h} p\right\rangle,\left\langle\mathbf{v}_{h}, q_{h}\right\rangle\right) \\
& +\varepsilon\left(\varphi_{h} \partial_{i, h} p_{h \varepsilon}, q_{h}\right), \quad\left\langle\mathbf{v}_{h}, q_{h}\right\rangle \in \mathscr{V}_{b h}(\mathscr{D}) .
\end{aligned}
$$

Then it follows by (5.6), the stability of $B$ on $\mathscr{V}_{b h}(\mathscr{D}) \times \mathscr{V}_{b h}(\mathscr{D})$, that

$$
\begin{aligned}
\|\left\langle\omega \partial_{i, h} \mathbf{u}-\mathbf{W}_{h},\right. & \left.\omega \partial_{i, h} p-R_{h}\right\rangle \|_{\mathscr{D}, 1,0} \\
\leqslant & \left(1+\frac{c_{0}}{c_{1}}\right) \inf _{\left\langle\mathbf{v}_{h}, q_{h}\right\rangle \in \mathscr{V}_{h h}(\mathscr{D})}\left\|\left\langle\omega \partial_{i, h} \mathbf{u}-\mathbf{v}_{h}, \omega \partial_{i, h} p-q_{h}\right\rangle\right\|_{\mathscr{D}, 1,0} \\
& +\frac{|\varepsilon|}{c_{1}}\left\|\partial_{i, h} p_{h \varepsilon}\right\|_{\mathscr{D}} \\
\leqslant & c h^{r}\|\langle\mathbf{u}, p\rangle\|_{r+2, r+1}+\frac{|\varepsilon|}{c_{1}}\left(\left\|\partial_{i, h}\left(p_{h \varepsilon}-p\right)\right\|_{\mathscr{D}}+\left\|\partial_{i, h} p\right\|_{\mathscr{D}}\right) \\
\leqslant & c h^{r}\|\langle\mathbf{u}, p\rangle\|_{r+2, r+1}+\frac{2|\varepsilon|}{c_{1} h}\|E\|+c|\varepsilon|\|p\| .
\end{aligned}
$$

We have, by the triangle inequality,

$$
\begin{aligned}
h\left\|\left\langle\partial_{i, h} \mathbf{e}, \partial_{i, h} E\right\rangle\right\|_{\mathscr{D}_{0}, 1,0} \leqslant & h\left\|\left\langle\partial_{i, h} \mathbf{u}-\mathbf{W}_{h}, \partial_{i, h} p-R_{h}\right\rangle\right\|_{\mathscr{D}_{0}, 1,0} \\
& +h\left\|\left\langle\mathbf{W}_{h}-\partial_{i, h} \mathbf{u}_{h \varepsilon}, R_{h}-\partial_{i, h} p_{h \varepsilon}\right\rangle\right\|_{\mathscr{D}_{0}, 1,0} \\
\leqslant & c\left(|\varepsilon| h\|\langle\mathbf{u}, p\rangle\|_{2,1}+|\varepsilon|^{2}\|p\|_{1}+h^{r+1}\|\langle\mathbf{u}, p\rangle\|_{r+2, r+1}\right) \\
& +h\left\|\left\langle\mathbf{W}_{h}-\partial_{i, h} \mathbf{u}_{h \varepsilon}, R_{h}-\partial_{i, h} p_{h \varepsilon}\right\rangle\right\|_{\mathscr{D}_{0}, 1,0} .
\end{aligned}
$$

Now

$$
B\left(\left\langle\mathbf{W}_{h}-\partial_{i, h} \mathbf{u}_{h \varepsilon}, R_{h}-\partial_{i, h} p_{h \varepsilon}\right\rangle,\left\langle\mathbf{v}_{h}, q_{h}\right\rangle\right)=0, \quad\left\langle\mathbf{v}_{h}, q_{h}\right\rangle \in \mathscr{V}_{h}\left(\mathscr{D}_{0}^{\prime}\right) .
$$

Thus, by Lemma 6,

$$
\begin{aligned}
\|\left\langle\mathbf{W}_{h}-\right. & \left.\partial_{i, h} \mathbf{u}_{h \varepsilon}, R_{h}-\partial_{i, h} p_{h \varepsilon}\right\rangle \|_{\mathscr{D}_{0}, 1,0} \\
\leqslant & c\left(h\left\|\left\langle\mathbf{W}_{h}-\partial_{i, h} \mathbf{u}_{h \varepsilon}, R_{h}-\partial_{i, h} p_{h \varepsilon}\right\rangle\right\|_{\mathscr{D}_{0}^{\prime}, 1,0}\right. \\
& \left.+\left|\left\langle\mathbf{W}_{h}-\partial_{i, h} \mathbf{u}_{h \varepsilon}, R_{h}-\partial_{i, h} p_{h \varepsilon}\right\rangle\right|_{\mathscr{D}_{0}^{\prime}, 0,-1}\right) \\
\leqslant & c h\left\|\left\langle\partial_{i, h} \mathbf{e}, \partial_{i, h} E\right\rangle\right\|_{\mathscr{D}_{0}^{\prime}, 1,0}+c\left|\left\langle\partial_{i, h} \mathbf{e}, \partial_{i, h} E\right\rangle\right|_{\mathscr{D}_{0}^{\prime}, 0,-1} \\
& +c h\left\|\left\langle\partial_{i, h} \mathbf{u}-\mathbf{W}_{h}, \partial_{i, h} R_{h}-\partial_{i, h} p\right\rangle\right\|_{\mathscr{D}_{0}^{\prime}, 1,0} \\
& +c\left|\left\langle\partial_{i, h} \mathbf{u}-\mathbf{W}_{h}, \partial_{i, h} R_{h}-\partial_{i, h} p\right\rangle\right|_{\mathscr{D}_{0}^{\prime}, 0,-1} \\
\leqslant & c\|\langle\mathbf{e}, E\rangle\|_{\mathscr{D}, 1,0}+c\left\|\left\langle\omega \partial_{i, h} \mathbf{u}-\mathbf{W}_{h}, \omega \partial_{i, h} p-R_{h}\right\rangle\right\|_{\mathscr{D}, 1,0} \\
\leqslant & c\left(h^{r}\|\langle\mathbf{u}, p\rangle\|_{r+2, r+1}+\frac{|\varepsilon|}{h}\|E\|+|\varepsilon|\|p\|_{1}\right) .
\end{aligned}
$$


Hence, we have that

$$
\begin{aligned}
& h\left\|\left\langle\mathbf{W}_{h}-\partial_{i, h} \mathbf{u}_{h \varepsilon}, R_{h}-\partial_{i, h} p_{h \varepsilon}\right\rangle\right\|_{\mathscr{D}_{0}, 1,0} \\
& \quad \leqslant c\left(|\varepsilon| h\|\langle\mathbf{u}, p\rangle\|_{2,1}+|\varepsilon|^{2}\|p\|_{1}+h^{r+1}\|\langle\mathbf{u}, p\rangle\|_{r+2, r+1}\right) .
\end{aligned}
$$

The result of Lemma 7, (5.26), now follows from (5.29) and (5.32).

We finally turn to the proof of Theorem 4. As we remarked earlier, since $\Omega^{\prime}$, $\Omega^{\prime} \subset \subset \tilde{\Omega} \subset \subset \Omega$, can be covered by a finite number of annuli, $\mathscr{D}_{0}$, which are compact in $\tilde{\Omega}$, we may assume that $\Omega^{\prime}=\mathscr{D}_{0}$ is an annulus. Now, since $\Omega^{\prime}=\mathscr{D}_{0} \subset \subset \tilde{\Omega}$, there is an annulus, $\mathscr{D}$, such that $\mathscr{D}_{0} \subset \subset \mathscr{D} \subset \subset \tilde{\Omega}$ and such that $\mathscr{D}_{0} \subset \subset \mathscr{D}$ are concentric. It then follows from (4.6) and (4.7) that

$$
\begin{aligned}
\left\|p-K * p_{h \varepsilon}\right\|_{\mathscr{D}_{0}} \leqslant & c\left(|\varepsilon| h\|\langle\mathbf{u}, p\rangle\|_{2,1}+|\varepsilon|^{2}\|p\|+h^{2}\|p\|_{2}+h^{r+1}\|\langle\mathbf{u}, p\rangle\|_{r+1, r}\right) \\
& +c \sum_{i=1}^{2}\left\|\partial_{i, h}\left(p-p_{h \varepsilon}\right)\right\|_{\mathscr{D},-1} .
\end{aligned}
$$

Now from (5.18) and Lemma 7 we have for $h$ sufficiently small,

$$
\left\|\partial_{i, h}\left(p-p_{h \varepsilon}\right)\right\|_{\mathscr{D},-1} \leqslant c\left(|\varepsilon| h\|\langle\mathbf{u}, p\rangle\|_{2,2}+|\varepsilon|^{2}\|p\|_{1}+h^{r+1}\|\langle\mathbf{u}, p\rangle\|_{r+2, r+1}\right) \text {. }
$$

Thus, from (5.33) and (5.34), we obtain

$$
\begin{aligned}
\| p & -K * p_{h \varepsilon} \|_{\mathscr{D}_{0}} \\
& \leqslant c\left(|\varepsilon| h\|\langle\mathbf{u}, p\rangle\|_{2,2}+|\varepsilon|^{2}\|p\|_{1}+h^{2}\|p\|_{2}+h^{r+1}\|\langle\mathbf{u}, p\rangle\|_{r+2, r+1}\right) .
\end{aligned}
$$

This completes the proof of Theorem 4.

Department of Chemical Engineering

and Materials Science

University of Minnesota

Minneapolis, Minnesota 55455

School of Mathematics

University of Minnesota

Minneapolis, Minnesota 55455

1. J. Bramble \& A. Schatz, "Higher order local accuracy by averaging in the finite element method," Math. Comp., v. 31, 1977, pp. 94-111.

2. P. G. Ciarlet, The Finite Element Method for Elliptic Problems, North-Holland, Amsterdam, 1978.

3. V. Girault \& P.-A. Raviart, Finite Element Approximation of the Navier-Stokes Equations, Lecture Notes in Math., Vol. 749, Springer-Verlag, Berlin, 1979.

4. T. Hughes, W. LiU \& A. Brooks, "Finite element analysis of incompressible viscous flows by the penalty function formulation," J. Comput. Phys., v. 30, 1979, pp. 1-60.

5. H. KHESHGI \& M. LUSKIN, "On the variable sign penalty approximation of the Navier-Stokes equation," Nonlinear Partial Differential Equations (Joel Smoller, ed.), Contemporary Mathematics, Vol. 17, Amer. Math. Soc., Providence, R. I., 1983.

6. H. KHESHGI \& L. SCRIVEN, "Finite element analysis of incompressible viscous flow by a variable penalty function method," Penalty-Finite Element Methods in Mechanics (J. N. Reddy, ed.), AMD-Vol. 51, A. S. M. E., New York, 1982.

7. H. KheShgi \& L. SCRIVEN, "Variable penalty method for finite element analysis of incompressible Stokes flow," Internat. J. Numer. Methods Fluids. (To appear.)

8. J. Nitsche \& A. Schatz, "Interior estimates for Ritz-Galerkin methods," Math. Comp., v. 28, 1974, pp. 937-958.

9. R. Temam, Navier-Stokes Equations, 2nd ed., North-Holland, Amsterdam, 1979. 